\title{
A Methodology for the Creation of Integrated Service Networks in Outpatient Internal Medicine
}

\author{
Miguel Angel Ortiz Barrios, Juan Escorcia Caballero, Fabián Sánchez Sánchez
}

\begin{abstract}
Extended patient waiting times for medical care in outpatient internal medicine has a direct impact on patient dissatisfaction. This issue has become increasingly relevant in Colombia where patient waiting times tend to be longer. In this context, a methodology based on value stream mapping (VSM) and collaborative sceneries has been created to examine different improving alternatives focused on the design of integrated service networks in outpatient internal medicine with the participation of two hospitals with mixed-patient type environment. First, an individual diagnosis for each hospital is made through VSM to identify non-value activities in the value chain and design improvement strategies for each process. Second, a strategic platform of the network is set. Third, communication and service protocols of the network are defined. Then, a simulation model is designed and validated to conduct experiments on the structure of the network. Finally, payment and risk tables are determined and key performance indexes of the network are established. The results prove the validity of the proposed approach upon reducing $75 \%$ of the lead time in this process creating a positive impact on population's health under satisfactory and equitable financial benefits for the participant hospitals.
\end{abstract}

Keywords

Integrated service networks, Value stream mapping, Collaborative sceneries, Outpatient internal medicine, Healthcare 